\title{
Aggregate-area radiative flux biases
}

\author{
Xuanji Wang, ${ }^{1}$ Jeffrey R. Key ${ }^{2}$ \\ ${ }^{1}$ Cooperative Institute for Meteorological Satellite Studies, University of Wisconsin-Madison, 1225 West Dayton Street, \\ Madison, WI 53706-1490, U.S.A. \\ ${ }^{2}$ Office of Research and Applications, National Environmental Satellite, Data, and Information Service, \\ National Oceanic and Atmospheric Administration, Madison, WI 53706, U.S.A.
}

\begin{abstract}
Most climate models treat surface and atmospheric properties as being horizontally homogeneous and compute surface radiative fluxes with average gridcell properties. In this study it is found that large biases can occur if sub-gridcell variability is ignored, where bias is defined as the difference between the average of fluxes computed at high resolution within a model cell and the flux computed with the average surface and cloud properties within the cell. Data from the AdvancedVery High Resolution Radiometer for the year-long Surface Heat Budget of the Arctic Ocean (SHEBA) experiment are used to determine biases in aggregate-area fluxes. A simple regression approach to correct for biases that result from horizontal variability was found to reduce the average radiative flux bias to near zero. The correction can be easily implemented in numerical models.
\end{abstract}

\section{INTRODUGTION}

Sea-ice and climate models have relatively coarse grids, typically on the order of $100-250 \mathrm{~km}$. In the computation of radiative fluxes, surface and atmospheric properties are treated as being horizontally homogeneous, and average gridcell properties are used, with either radiative transfer models or simple paramerization schemes (cf. Key and others, 1996). Recent studies have shown that using areaaverage properties can result in errors in estimating surface radiative and turbulent fluxes because of non-linear relationships (Liou and Wittman, 1979; Mahrt, 1987; Sellers, 1991; Key, 1993; Marshak and others, 1995; Friedl, 1996). How large are the errors in radiative fluxes computed from areaaverage surface and cloud properties? The magnitude of the errors depends on the degree of non-linearity in the relationship and on the spatial variability of the parameters.

The purposes of this paper are to (a) evaluate the biases in downwelling shortwave and longwave radiative fluxes calculated from area-average surface and cloud properties vs averages of fluxes calculated at a relatively high spatial resolution, and (b) develop a simple regression approach for correcting the biases. Satellite data are used to assess the relative magnitude of the radiative flux biases. The objective is to determine the importance of parameterizing sub-gridcell variability in sea-ice and climate models.

\section{DEFINITION, DATA AND FLUX GALGULATIONS}

In this study radiative fluxes for a hypothetical model gridcell are determined in two ways: (1) by calculating the fluxes with average surface and cloud properties over the gridcell, analogous to what is done in models, and (2) by calculating fluxes for every $5 \times 5 \mathrm{~km}^{2}$ satellite pixel within the gridcell, and then averaging those fluxes over the cell. In both cases the surface and cloud properties are estimated from satellite data. We call the former method the "area-average" method, and the latter the "pixel-average" method. The pixel-average method is similar to the concept of the "independent pixel approximation" used in studies of three-dimensional radiative transfer effects (cf. Marshak and others, 1998).

Data from the Advanced Very High Resolution Radiometer (AVHRR), on board U.S. National Oceanic and Atmospheric Administration polar-orbiting satellites, are used in this study. The specific dataset is a product of the AVHRR Polar Pathfinder (APP) project (M. F. Meier and others, http://ams.allenpress.com/amsonline/?request=getdocument $\&$ issn $=10872-562 \&$ volume $=001 \&$ issue $=05 \&$ page $=$ 0001; Maslanik and others, 1997, 1999, 2001). The APP data are twice-daily composites available at $5 \times 5 \mathrm{~km}^{2}$ pixel size for June 1981-1998. The study period September 1997August 1998 and the area (Fig. 1) correspond to the Surface Heat Budget of the Arctic Ocean (SHEBA) field experiment, where an ice-breaker drifted with the pack ice for 1 year (Moritz and others, 1993). The APP standard products are clear-sky surface temperature and broadband albedo, a cloud mask, sea-ice motion and the calibrated, geolocated channel data and viewing/illumination geometry. We have extended this product set to include all-sky surface skin temperature and broadband albedo, cloud properties (particle phase, effective radius, optical depth, temperature and pressure) and radiative fluxes. Algorithms in the Cloud and Surface Parameter Retrieval (CASPR) system are employed. Radiative fluxes are computed in CASPR using FluxNet (Key and Schweiger, 1998). See Key (2001) and references therein for more information on the algorithms and their validation.

\section{SPATIAL VARIABILITY AND ANALYSIS}

Semivariance analysis is employed to describe the spatial variability of the surface radiative fluxes, cloud and surface properties. The semivariogram is a structure function that, like autocorrelation, describes the correspondence between 


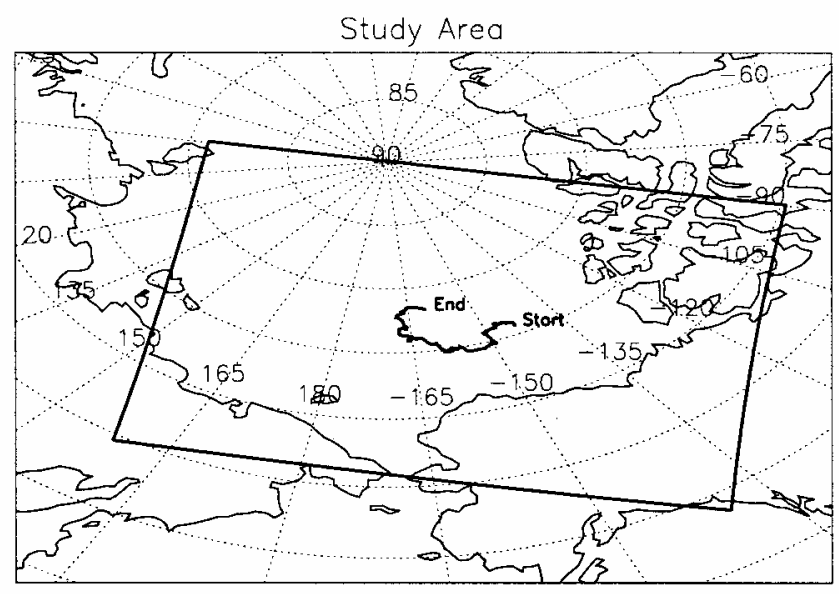

Fig. 1. The study area. The curve is the drift track of the SHEBA ship during the year-long SHEBA experiment. It started at $75.70^{\circ} \mathcal{N}, 144.10^{\circ} \mathrm{W}$ on 2 October 1997, and ended at $78.20^{\circ} \mathcal{N}, 160.70^{\circ}$ Won 3 August 1998.

observations made at some distance. Therefore, a parameter measured at one location provides some information about the parameter at another location. The semivariance $r(h)$ for a distance lag $h$ is defined as:

$$
r(h)=\frac{1}{2 N} \sum_{i=1}^{N}\left[\phi\left(x_{i}\right)-\phi\left(x_{i}+h\right)\right]^{2},
$$

where $x$ is a location and $\phi$ describes the data (e.g. albedo or temperature). The change in semivariance with lag illustrates how rapidly the autocorrelation changes, while the magnitude of the semivariance indicates the degree of variability. One feature of the semivariogram is the so-called "sill". The sill is the ordinate value at which the semivariogram reaches a maximum and becomes asymptotic, indicating that beyond that distance variance does not increase substantially. That distance beyond which no significant correlation exists is called the "range".

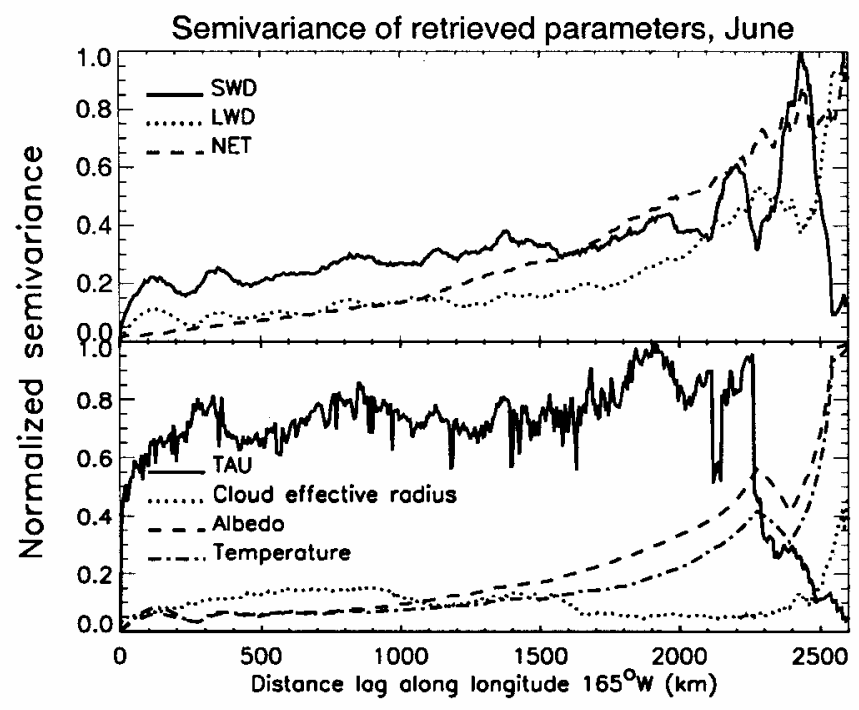

Fig. 2. Space-lag semivariogram for SWD and LWD fluxes, net radiation flux (NET) at the surface, cloud optical depth ( TAU), surface broadband albedo, surface skin temperature and cloud effective radius. The semivariance of each parameter was normalized by the maximum semivariance. Monthly means along longitude $165^{\circ} \mathrm{W}$ were used.

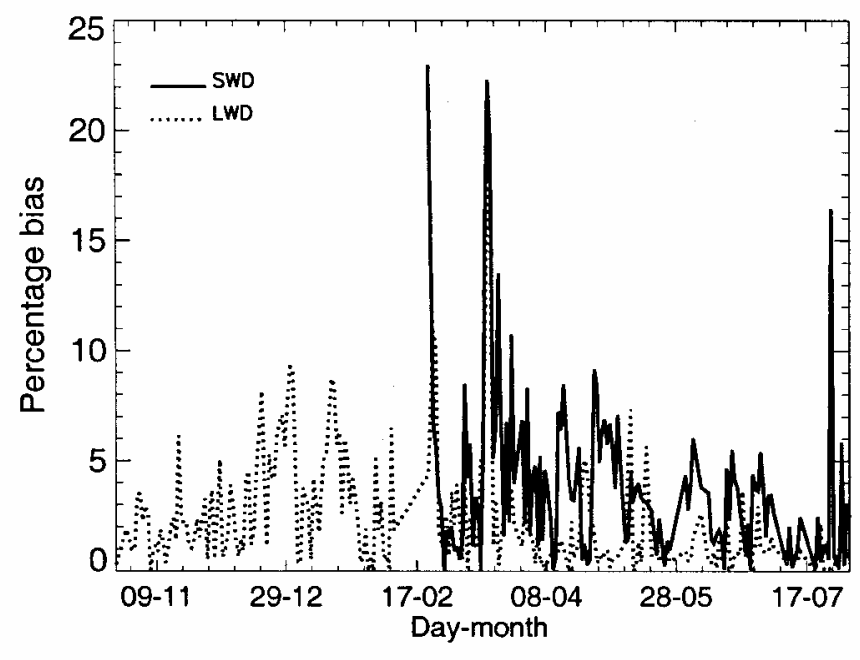

Fig. 3. Percentage biases of SWD and LWD fluxes. The bias is defined as area-average flux minus pixel-average flux, so the percentage bias is equal to bias divided by the pixel-average flux. The solid line is SWD, and dotted line is LWD for area size $505 \times 505 \mathrm{~km}^{2}$. Biases for other cell sizes are similar.

Figure 2 shows the spatial semivariogram of surface and cloud properties for June 1998 based on monthly mean AVHRR-derived values along the longitude $165^{\circ} \mathrm{W}$. The normalized semivariance shown in the figure is the semivariance at each lag divided by the maximum semivariance. Cloud fraction is not shown because cloud fraction can only be 0 or 1, i.e. clear or overcast for the $5 \times 5 \mathrm{~km}^{2}$ pixels. Those pixels were used to calculate the spatial semivariogram, so it is not meaningful to calculate cloud-fraction semivariogram based on only two cases. In the spatial semivariogram, radiative fluxes, surface skin temperature and surface broadband albedo show persistence with distance. They have two ranges corresponding to two different scales: about $150 \mathrm{~km}$ and $>2000 \mathrm{~km}$. This is reasonable because the semivariogram is calculated along the longitude $165^{\circ} \mathrm{W}$, the latter range reflecting the large-scale systematic latitudinal distribution of the climate, and the former reflecting the mesoscale climatic variation. Cloud-particle effective radius and optical depth have a range of about $400 \mathrm{~km}$, which means that within that distance the cloud properties are correlated.

The gradual increase in the normalized semivariance of surface skin temperature and broadband albedo relative to that of cloud optical depth and particle effective radius implies that the spatial variance increases much less rapidly for surface properties than for cloud properties. It clearly shows that most parameters change with spatial scale in a non-linear way. The semivariance pattern may be different in other seasons, so Figure 2 is only one description of spatial variability. Bias corrections discussed below are based on data from all seasons, so the seasonal differences are accounted for implicitly. The spatial and temporal variability of surface and cloud properties is discussed in detail in Wang and Key (2001).

We expect that the radiative fluxes calculated with the area-average and pixel-average methods will be different because (a) surface and cloud parameters exhibit spatial variability on scales less than that of a typical climate- or ice-model gridcell, (b) the magnitude of the variability differs for each parameter, and (c) the relationship between some parameters and the fluxes is non-linear. To test that hypothesis, both methods were used to compute the flux 

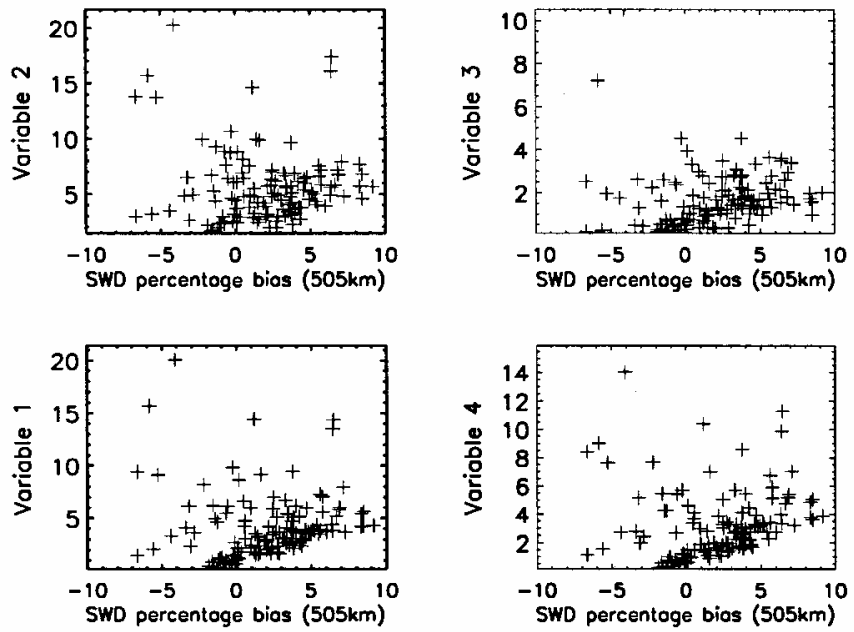

Fig. 4. The relationship between the parameters used in the regression equation for the $S W D$ flux-bias correction and the SWD flux percentage biases. Variable 1 is $C \tau$ ( $C$ is cloud fraction, and $\tau$ is cloud optical depth), variable 2 is $\tau$, variable 3 is $C \tau \mu$ ( $\mu$ is cosine of the solar zenith angle) and variable 4 is $C \tau \alpha$ ( $\alpha$ is surface broadband albedo).

biases in the downwelling shortwave (SWD) and downwelling long wave (LWD) radiative flux calculations, where the bias is defined as the area-average flux minus the pixelaverage flux. Figure 3 shows the biases as percentages for the SWD and LWD radiative fluxes. The bias can be as large as $22 \%$ for both fluxes, indicating that the correction should be taken into account when average surface and cloud properties are used to calculate model gridcell fluxes.

The relationship between the biases and geophysical parameters is analyzed using multivariate regression analysis (Liou and Wittman, 1979) of the form

$$
\begin{gathered}
\operatorname{SWDBias}(C, \tau, \mu, \alpha)=\sum_{i=0}^{3} \sum_{j=0}^{3} \sum_{k=0}^{3} \sum_{l=0}^{3} b_{i j k l} C^{i} \tau^{j} \mu^{k} \alpha^{l} \\
\operatorname{LWDBias}(C, T)=\sum_{i=0}^{3} \sum_{j=0}^{3} b_{i j} C^{i} T^{j}
\end{gathered}
$$

where SWDBias and LWDBias denote the SWD and LWD flux biases, respectively, $b_{i j k l}$ and $b_{i j}$ are the regression coefficients for the SWD and LWD flux-bias correction, $C$ is cloud fraction, $\tau$ is cloud optical depth, $\mu$ is the cosine of the solar zenith angle, $\alpha$ is surface broadband albedo and $T$ is surface skin temperature. Examinations of all surface and cloud properties revealed that combinations of cloud fraction, cloud optical depth, surface broadband albedo and solar zenith angle for SWD flux biases influence the magnitude of the biases to a much greater degree than other parameters (Fig. 4). For the LWD flux biases the cloud fraction, surface skin temperature and their combination contribute the most to the explanation of bias variance (Fig. 5).

For SWD fluxes, the biases tend to change from negative to positive when the cloud optical depth becomes large. When the cloud fraction is small and/or surface skin temperature is low, the LWD biases are negative. With the increases of the cloud fraction and/or temperature the LWD flux-bias changes become positive. Based on these relationships, the five parameters used to estimate the SWD biases are cloud fraction, cloud optical depth, the product of cloud fraction and optical depth, the products of cloud fraction, optical
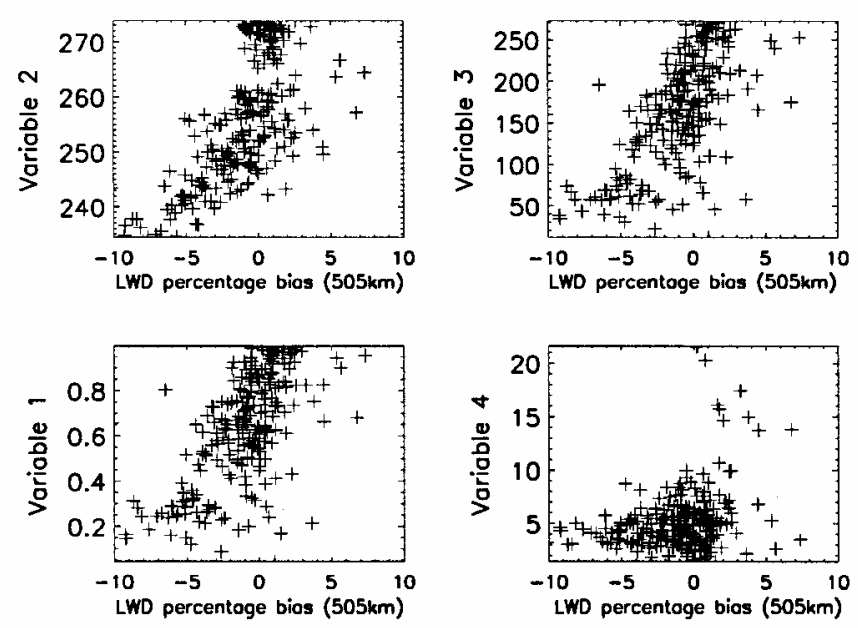

Fig. 5. The relationship between the parameters used in the regression equation for the LWD flux-bias correction and the LWD flux percentage biases. Variable 1 is $C$ ( $C$ is cloud fraction), variable 2 is $T$ ( $T$ is surface skin temperature) and variable 3 is $C T$.

depth, the cosine of solar zenith angle and surface broadband albedo. The multiple correlation coefficient for the five parameters used to estimate SWD flux biases is 0.63 . For the LWD flux biases, three parameters are used: cloud fraction, surface skin temperature and the product of cloud fraction and surface skin temperature. The multiple correlation coefficient for the LWD flux-bias estimation is 0.62 .

\section{BIAS GORREGTIONS}

The analysis and correlation imply that it may be possible to correct the radiative fluxes for errors resulting from subgridcell variability. The regression analysis was performed on the SHEBA AVHRR retrievals. Regression equations were developed to estimate the SWD and LWD flux biases under all weather conditions, but with only those terms in Equations (2) and (3) that passed the statistical significance

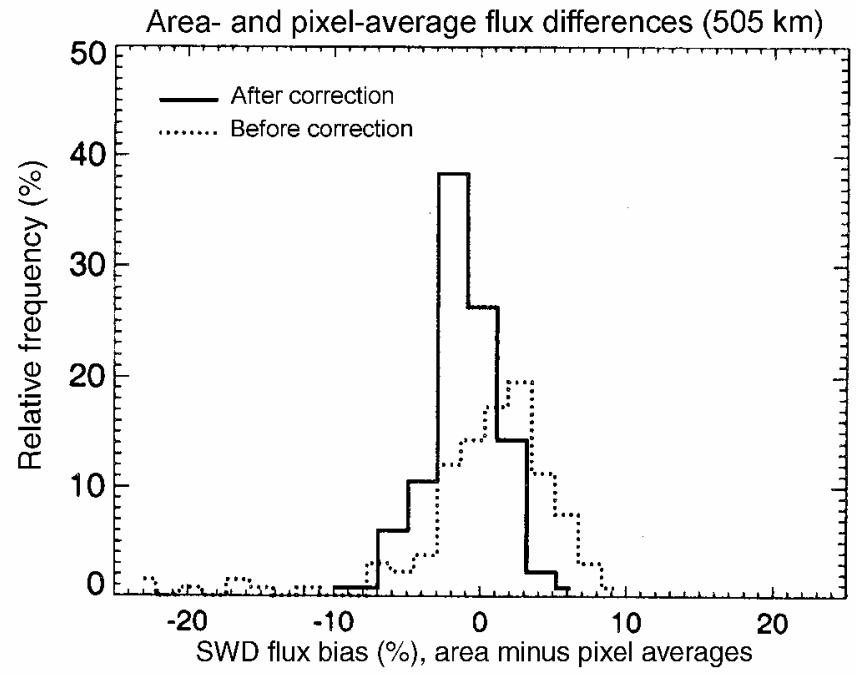

Fig. 6. Relative frequency of SWD flux biases between the areaaverage and pixel-average fluxes before and after correction. Values shown are for the period September 1997-August 1998. 


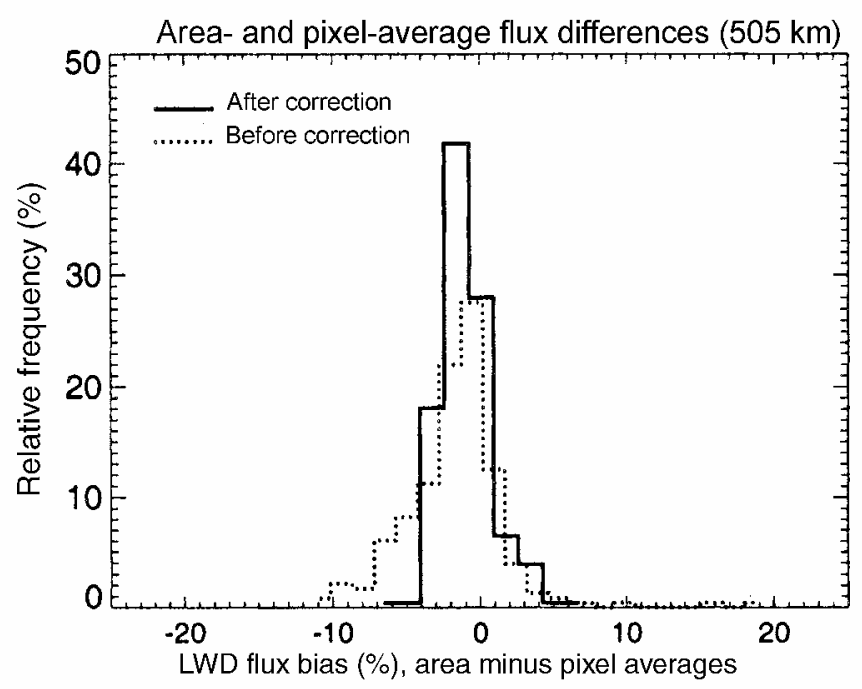

Fig. 7. Relative frequency of $L W D$ flux biases between the areaaverage and pixel-average fluxes before and after correction. Values shown are for the period September 1997-August 1998.

test (at a significance level of 0.05). The regression equations are:

$$
\begin{aligned}
\text { SWDBias }= & -5.6519+0.33793 \tau+18.246 C-3.9539 C \tau \\
& +3.3107 C \tau \mu+2.1057 C \tau \alpha
\end{aligned}
$$

$\mathrm{LWDBias}=-77.403+73.115 C+0.28325 T-0.26119 C T$.

Figures 6 and 7 show the uncorrected and corrected bias frequency distributions. The bias correction equations are applicable to all 14 hypothetical model gridcell sizes, from $15 \times 15 \mathrm{~km}^{2}$ to $505 \times 505 \mathrm{~km}^{2}$. This may be because the dependence of the flux biases on spatial scale is only significant at scales much smaller than those examined here. For example, aircraft studies have shown that the "radiative smoothing scale" is on the order of $200-300 \mathrm{~m}$ for marine stratocumulus (Marshak and others, 1997). The results show that the overall mean biases for SWD and LWD fluxes are small, but the standard deviations of SWD and LWD flux biases are 14.40 and $6.25 \mathrm{~W} \mathrm{~m}^{-2}$, respectively, before the correction. After the correction the overall standard deviations are reduced to 8.84 and $3.57 \mathrm{~W} \mathrm{~m}^{-2}$ for SWD and LWD flux biases. The largest biases generally occur in the spring and fall, when surface characteristics change most rapidly.

\section{GONGLUSION}

Satellite retrievals of surface, cloud and radiation parameters over sea ice were used to investigate their spatial variability in the western Arctic during the SHEBA experiment. The spatial variance increases much less rapidly for surface properties (temperature and albedo) than for cloud properties (optical depth and particle effective radius in particular). Given the spatial variability of surface and cloud parameters and their non-linear relationship with radiative fluxes, one would expect that using mean surface and cloud properties within a climate- or ice-model gridcell to compute radiative fluxes could result in substantial errors. If so, biases in calculated radiative fluxes could influence modeled latent- and sensible-heat fluxes, and ultimately sea-ice growth and melt.

This was investigated by computing average radiative fluxes for 14 hypothetical model gridcell sizes as (a) the mean of the fluxes calculated for every $5 \times 5 \mathrm{~km}^{2}$ pixel within the cell, and (b) the result of using area-average mean surface and cloud properties in the flux calculation. The bias can be as large as $22 \%$ for both the SWD and LWD fluxes, indicating that corrections should be made when areaaverage properties are used to calculate model gridcell fluxes. Other radiative transfer models and parameterizations should provide similar results unless they are tuned to radiative flux observations, which inherently account for small-scale variability. Additionally, because the same method of computation of radiative fluxes was used to compute both pixel-average and area-average fluxes, any systematic error in the method would be removed.

A simple regression approach to correcting the fluxes for the biases that result from horizontal variability was found to reduce the average biases to nearly zero and to reduce the standard deviations by $50 \%$. The correction can be easily implemented in numerical models. The bias-correction algorithm should be applied to the models with model gridscale larger than $15 \times 15 \mathrm{~km}^{2}$.

Can the corrections proposed here be applied to regions outside of the Arctic (e.g. the tropics)? The answer depends on how strongly the differences in frequency and spatial distributions of cloud and surface parameters influence the flux biases. Another important issue is how universal the correction method is to individual Arctic seasons. It is important to see how the correction method behaves seasonally, to what extent the method can correct the flux biases for each of seasons and what might be the dominant mechanisms to control the flux biases in different seasons. These issues are under investigation.

\section{ACKNOWLEDGEMENTS}

This research was supported by NASA grants NAG5-8625 and NAG5-6666, and U.S. National Science Foundation grant OPP-0096085. We thank C. Fowler, J. Maslanik, T. Scambos and T. Haran for all their work on the APP dataset. We also thank the reviewers for their thoughtful questions and useful suggestions on the manuscript.

\section{REFERENGES}

Friedl, M. A. 1996. Relationships among remotely sensed data, surface energy balance, and area-averaged fluxes over partially vegetated land surfaces. f. Appl. Meteorol., 35(11), 2091-2103.

Key, J. R. 1993. Estimating the area fraction of geophysical fields from measurements along a transect. IEEE Trans. Geosci. Remote Sensing, GE31(5), 1099-1102.

Key, J. R. 2001. The cloud and surface parameter retrieval (CASPR) system for polar AVHRR. Wisconsin, MA, Space Science and Engineering Center.

Key, J. and A. J. Schweiger. 1998. Tools for atmospheric radiative transfer: Streamer and FluxNet. Comput. Geosci., 24(5), 443-451.

Key, J. R., R. A. Silcox and R. S. Stone. 1996. Evaluation of surface radiative flux parameterizations for use in sea ice models. F. Geophys. Res., 101(C2), 3839-3849.

Liou, K. and G. D. Wittman. 1979. Parameterization of the radiative properties of clouds. 7. Atmos. Sci., 36(7), 1261-1273.

Mahrt, L. 1987. Grid-averaged surface fluxes. Mon. Weather Rev., 115(8), $1550-1560$.

Marshak, A., S. Davis, R. F. Cahalan, W. Wiscombe and R. Cahalan. 1995 Radiative smoothing in fractal clouds. 7. Geophys. Res., 100(D12), 26,247-26,261.

Marshak, A., S. Davis, W. Wiscombe and R. Cahalan. 1997. Inhomogeneity effects on cloud shortwave absorption measurements: two-aircraft simulation. F. Geophys. Res., 102(D4), 16,619-16,637.

Marshak, A., S. Davis, R. F. Cahalan and W. Wiscombe. 1998. Nonlocal 
independent pixel approximation: direct and inverse problems. IEEE Trans. Geosci. Remote Sensing, GE-36(1), 192-205.

Maslanik, J., C. Fowler, J. Key, T. Scambos, T. Hutchinson andW. Emery. 1997. AVHRR-based Polar Pathfinder products for modeling applications. Ann. Glaciol., 25, 388-392.

Maslanik, J., A. Lynch and C. Fowler. 1999. Assessing 2-D and coupled-model simulations of sea ice anomalies using remotely-sensed polar pathfinder products. In Fifth Conference on Polar Meteorology and Oceanography, 10-15 January 1999, Dallas, Texas. Proceedings. Boston, MA, American Meteorological Society, 476-479.

Maslanik, J. A., J. Key, C.W. Fowler, T. Nguyen and X. Wang. 2001. Spatial and temporal variability of satellite-derived cloud and surface characteristics during FIRE-ACE. 7. Geophys. Res., 106(D14), 15,233-15,249.

Moritz, R. E., J. A. Curry, A. S. Thorndike and N. Untersteiner. 1993. Surface heat budget of the Arctic Ocean. Seattle, WA, University of Washington. Polar Science Center. Applied Physics Laboratory. SHEBA Project Office. (ARCSS/OAII Tech. Rep 3.)

Sellers, P. 1991. Modeling and observing land-surface--atmosphere interactions on large scales. Surveys in Geophysics, 21 (1), 85-114.

Wang, X. and J. R. Key. 2001. Spatial variability of the sea-ice radiation budget and its effect on aggregate-area fluxes. Ann. Glaciol., 33, 248-252. 